\title{
Metformin Targets Glucose Metabolism in Triple Negative Breast Cancer
}

\section{Wahdan-Alaswad RS, Edgerton SM, Salem HS, and Thor AD}

Department of Pathology, School of Medicine, University of Colorado, Anschutz Medical Campus, Aurora, USA

"Corresponding author: Ann D Thor, Department of Pathology, School of Medicine, University of Colorado, Anschutz Medical Campus, Aurora, USA, Tel: 303-724-3704; E-mail: ann.thor@ucdenver.edu

Received date: March 04, 2018; Accepted date: March 14, 2018; Published date: March 21, 2018

Copyright: () 2018 Wahdan-Alaswad RS, et al. This is an open-access article distributed under the terms of the Creative Commons Attribution License, which permits unrestricted use, distribution, and reproduction in any medium, provided the original author and source are credited.

\begin{abstract}
Metformin is the most widely administered anti-diabetic agent worldwide. In patients receiving metformin for metabolic syndrome or diabetes, it reduces the incidence and improves the survival of breast cancer (BC) patients. We have previously shown that metformin is particularly potent against triple negative breast cancer (TNBC), with a reduction of proliferation, oncogenicity and motility, inhibition of pro-oncogenic signaling pathways and induction of apoptosis. These BCs are well recognized to be highly dependent on glucose/glucosamine (metabolized through anaerobic glycolysis) and lipids, which are metabolized for the production of energy and cellular building blocks to sustain a high rate of proliferation. We have previously demonstrated that metformin inhibits lipid metabolism, specifically targeting fatty acid synthase (FASN), cholesterol biosynthesis and GM1 lipid rafts in TNBC. We also reported that glucose promotes phenotypic aggression and reduces metformin efficacy. We now show that metformin inhibits several key enzymes requisite to glucose metabolism in TNBC, providing additional insight into why metformin is especially toxic to this subtype of BC. Our data suggests that the use of metformin to target key metabolic defects in lipid and carbohydrate metabolism in cancer may be broadly applicable, especially against highly aggressive malignant cells.
\end{abstract}

Keywords Breast cancer; Metformin; Glucose metabolism; Triple negative breast cancer; Glycolysis

\section{Introduction}

Breast cancer (BC) morbidity and mortality remain stubbornly high worldwide, despite the fact that disease characteristics vary by geography, ethnicity, age, body mass and other factors. While the prognosis for BC patients is better in the US than in much of the world, our incidence of disease is especially high in women with obesity and type II diabetes/metabolic syndrome. Experts anticipate that in 2018,266,000 new invasive and 64,000 new in situ breast cancer (BC) cases will be diagnosed in the US [1]. The majority of these BCs will be hormone receptor-positive (i.e. express oestrogen (ER) and/or progesterone receptor (PR)), responsive to surgery and endocrine therapy (for low risk cases), and have a good prognosis [2]. In contrast, the least common BC known as triple negative breast cancer (TNBC) lacks ER, PR and a tyrosine kinase receptor HER2, is highly aggressive and associated with the worst outcome [3-5].

Gender (being female) is the most potent risk factor for BC [6]. A family history of $\mathrm{BC}$ (typically involving first-degree relatives at a young age) is also an important risk factor. Heritable genetic alterations are associated with approximately one-tenth of all cases of $\mathrm{BC}$ [7]. Other important risk factors include age, reproductive and menstrual history, a lack of physical activity, obesity and type II diabetes [7]. For all women with both obesity and type II diabetes, the risk of $\mathrm{BC}$ increases by as much as $20 \%$ [8]. Less well-appreciated, gestational diabetes, pre-diabetes (the so-called metabolic syndrome) or a family history of diabetes also enhances a woman's risk for BC $[8,9]$. The impact of obesity on BC risk is also influenced by age and ethnicity [10]. For example, obesity is not a strong risk factor for premenopausal Caucasian females. In older Caucasian women, however, both obesity and type II diabetes increase the risk of hormone receptor-positive BC significantly [11]. These chronic diseases are also independently associated with a worse prognosis and higher diseaseassociated mortality for these women $[11,12]$. In contrast, obesity is significantly associated with an increase in BC risk in young (premenopausal) African American (AA) women [13-15]. These women most often develop TNBC, a subtype that is usually resistant to standard chemotherapy and targeted therapeutics $[11,16,17]$.

A historically attributed mechanism by which obesity (predominantly in a central, or abdominal distribution) promotes BC is the peripheral conversion of testosterone in adipocytes, leading to increases in circulating, bioavailable oestrogen (particularly problematic in post-menopausal women) [18]. More recent studies have shown that abdominal obesity influences BC development and outcomes through other mechanisms as well; including: systemic shifts in carbohydrate and fat metabolism, up regulation of pro-carcinogenic factors such as cytokines and growth factors (like insulin and insulinlike growth factors), modulation of the immune system and macrophage activation, as well as other systemic effects reviewed in details elsewhere [19-21]. Of note, obesity is often associated with the development of pre-diabetes (the so-called metabolic syndrome) or type II diabetes. Thus, dysregulation of carbohydrate and lipid metabolism often occurs together, typically prior to the development of BC.

With transformation of benign breast epithelial cells to the malignant phenotype, significant changes in fat metabolism and intracytoplasmic fat accumulation are often observed (particularly in TNBC). In fact, all cancer subtypes have shown an enhanced reliance on de novo fatty acid biosynthesis [22], irrespective of the availability of extracellular lipid derived from diet or adipose storage $[23,24]$. This so-called 'lipid switch' and the importance of Acetyl-CoA-carboxylase alpha to the malignant phenotype of $\mathrm{BC}$ cells have been well described by others $[25,26]$. We have previously shown that the anti-diabetic 
Page 2 of 6

drug metformin has potent action against these shifts in lipid metabolism. More specifically metformin targets critical components of fatty acid synthesis [27] as well as cholesterol biosynthesis, resulting in shifts in GM1 lipid rafts and associated receptor signaling [28]. The focus of this report is to provide new data regarding the effects of metformin on carbohydrate metabolism, another critical component of malignant cell metabolism that is requisite for cancer cell survival, proliferation and progression.

\section{Metabolic Syndrome, Breast Cancer and Dysregulation of Carbohydrate Metabolism in Cancer}

Metabolic syndrome and type II diabetes are associated with systemic dysregulation of lipid and carbohydrate metabolism. These changes disrupt a broad array of cell types and put the patient at an increased risk of cardiovascular disease and cancer [29]. Both type II diabetes and metabolic syndrome are independent risk factors for BC [30-32], although the complex mechanism by which this occurs in the breast is not well understood. The increase in serum insulin/insulin resistance and insulin-like growth factor associated with these disorders is one likely mechanism, as they are associated with an increase in breast cancer incidence and a worse prognosis [33-37]. Metabolic dysregulation is also associated with an increase in serum glucose and other energy precursors such as fructose and glucosamine that can be metabolized to adenosine triphosphate (ATP) and other factors to facilitate cancer replication and tumor growth even in a hypoxic environment [38].

Dysregulation of carbohydrate metabolism to preferentially use aerobic glycolysis is a well-recognized hallmark of cancer [39]. This metabolic reprogramming is achieved through a complex interplay of regulatory networks involving: phosphatidylinositide 3-kinase (PI3K), protein kinase B (Akt), mammalian target of rapamycin (mTOR), phosphatase and tensin homolog (PTEN), and 5' AMP-activated protein kinase (AMPK) [40,41]. Alternative oncogenic mechanisms involving c-Myc [42], hypoxia-inducible factor 1-alpha (HIF1a) [43], epidermal growth factor receptor (EGFR), tumor protein 53 (P53) and the Met receptor have also been implicated in the transformative process by which cancer cells switch to aerobic glycolysis [44-47].

\section{Alterations of Glucose Metabolism in Breast Cancer}

In order to meet the need for increased glucose intake from extracellular sources, cancer cells frequently upregulate membrane associated glucose transport proteins known as GLUTs [48-50], as well as associated cofactors (e.g.SGLT1) that can facilitate this process [51]. Of the various GLUT family members, GLUT1 and GLUT3 are the most highly expressed in BCs [52]. GLUT1 (SLC2A1) upregulation has been frequently reported in studies of TNBC, where it has been associated with a worse prognosis and treatment resistance [53]. In a preclinical model, GLUT1 also appears to be requisite to HER2 induced mammary tumorigenesis [54]. Other processes associated with carbohydrate metabolism have also been shown to be altered in TNBC, including oxidative phosphorylation [55] and glycolytic flux [56]. Thus, alterations of glucose metabolism are frequent, arise from multiple mechanisms and drive breast carcinogenesis (see elsewhere for a more extensive discussion [57]).

\section{Glucose Metabolism and Mechanisms of Metformin Action}

We previously reported that glucose promotes phenotypic cancer aggression and reduces the efficacy of metformin in all molecular subtypes of BC [58]. We have shown that the mechanisms of metformin action vary by molecular subtype of the disease [58-61], and that TNBCs are especially sensitive to its anti-cancer effects. More specifically, metformin blocks cellular proliferation, reduces oncogenicity, targets stem cells, slows motility, and induces apoptosis in TNBC [58-61]. More recently we have studied the effects of metformin on mammary tumors that arise in obese vs. lean rats as well as obese overfed mice. Each of these models was used to investigate the role of metabolic dysregulation associated with obesity and carbohydrate dysregulation on mammary tumor development, progression and metformin efficacy $[54,62,63]$. Using these and other in vitro models, we seek to define mechanisms of metformin action at the subcellular level.

Metformin reduces insulin resistance, promotes glucose and lipid homeostasis, particularly in liver and skeletal muscle. The most widely recognized mechanism of metformin action is through inhibition of the mammalian target of rapamycin complex I (mTORC1) in both AMPK-dependent and independent processes [64]. Less is known about its effects on benign or malignant breast epithelial cells. We have shown that metformin attenuates a number of specific oncogenic signaling pathways in BC not widely studied in other organ systems, including: STAT3 [64], transforming growth factor- $\beta$ (TGF- $\beta$ ) mediated activation of Smad2/Smad3 and ID1 [65], and the organic cation transporter (OCT1) [62]. We have also reported diverse targets of metformin to disrupt the aberrant lipid metabolism in BC cells. It attenuates de novo fatty acid synthesis through down-regulation of fatty acid synthase (FASN), via up-regulation of the microRNA 193b [27]. Metformin also inhibits 26 steps in the cholesterol synthesis pathway; resulting in a reduction of GM1 lipid raft generation and stability, as well as EGFR signaling [28].

\section{Metformin Attenuates Key Genes involved in Glucose Metabolism in TNBC}

We have previously shown that glucose promotes $\mathrm{BC}$ aggression and reduces metformin efficacy in vitro [58]. Using a carcinogen-induced rodent model of mammary tumorigenesis, we have also reported that overfed obese animals with the equivalent of metabolic syndrome (defined by elevated serum glucose) showed a $50 \%$ increase in glucose uptake by their mammary tumor cells, associated with enhanced proliferation and metabolic "reprogramming" similar to what was observed in human $\mathrm{BC}$ cells in vitro[63]

Furthermore, we have shown that metformin had significant antitumor effects in this rodent model. These findings suggest a basis for the epidemiological and data indicating that in patients with metabolic syndrome or type II diabetes, metformin treatment reduces cancer incidence and improves survival for patients that develop the disease (in stark contrast to other anti-cancer agents including insulin that increases $\mathrm{BC}$ risk) [34-36]. We postulate that metformin is especially potent against TNBC because of its enhanced dependence on glucose (mediated through SLC2A1) and glutamine (mediated through SLC6A14) and its markedly aberrant mitochondrial respiration [45].

Given that one of the primary metabolic changes observed in $\mathrm{BC}$ tumorigenesis is a marked increase in catabolic glucose metabolism, 
Page 3 of 6

and metformin's ability to disrupt these shifts, we sought to identify direct targets of the drug amongst glucose transporters and key enzymes of the glycolytic pathway. In brief, TNBC cells MDA-MB-468 and MDA-MB-231 were grown in either $17 \mathrm{mM}$ or $5 \mathrm{mM}$ glucose, with or without metformin. Purified mRNA was used for gene expression profiling [27, 58]. Data is shown in Figure 1. Metformin significantly down-regulated a number of glucose transporter proteins including: GLUT1 (SLC2A1), GLUT10 (SLC2A10), GLUT12 (SLC2A12), GLUT14 (SLC6A14), and Glucose-6-Phosphate transporter (SLC37A4) in MDA-MB-468 TNBC cells. Of these, GLUT1's downregulation by metformin is likely to have the most translational value in patients with TNBC, as it has been shown to be the predominant glucose transporter in this molecular subtype, and its expression has been associated with a worse survival. Metformin was shown to upregulate only one glucose transporter, GLUT12.

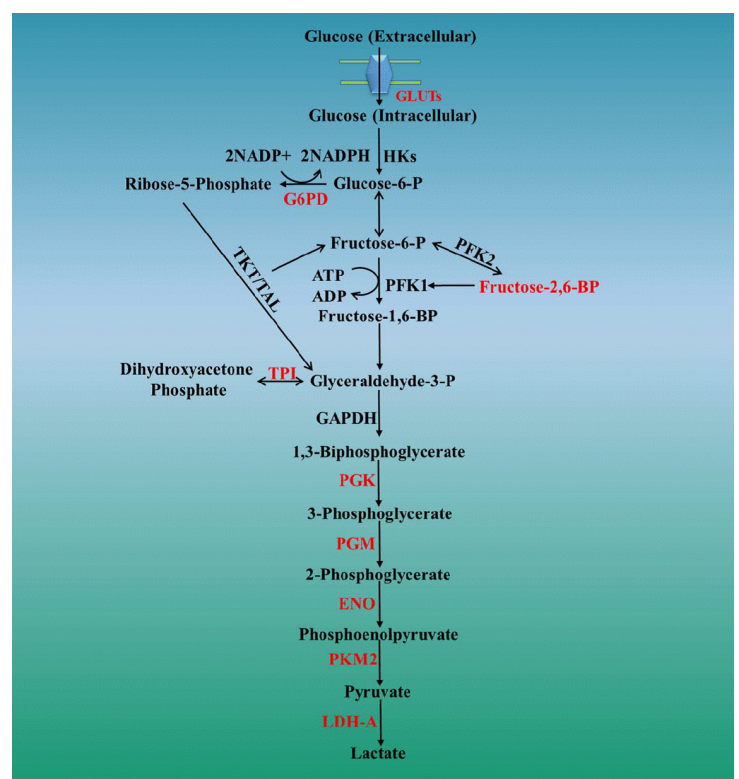

Figure 1: Metformin Attenuates Key Genes involved in Glucose Metabolism in TNBC.
In this experiment, we also showed that metformin attenuated the expression of a number of important genes involved in glucose metabolism. Over 20 genes were shown to be down-regulated, see Table 1 and Figure 1 (metformin target genes in red). At both low and high concentrations of glucose in the culture media (data from $17 \mathrm{mM}$ glucose not shown but similar), metformin down-regulated G6PD and triose phosphate isomerase (TPI), a key enzyme that participates in converting glyceraldehyde-3-P to dihydroxyacetone phosphate. Additionally, metformin targeted phosphoglycerate kinase 1 (PGK), phosphoglucomutase 1 (PGM), enolase 1 (alpha) ENO, pyruvate kinase muscle 2 (PKM2), and lactate dehydrogenase A (LDHA). Of note, metformin enhanced transcription of phosphoglucomutase 5 (PGM5), phosphoglucomutase 5 pseudogene 2 (PGM5P2), aldo-keto reductase family 1 Member B10 (AKR1B10), aldo-keto reductase family 1 member C2 (AKR1C2), and pyruvate dehydrogenase kinase 4 (PDK4).

Metformin also inhibited lactose dehydrogenase (LDH). LDH functions downstream of hexokinase $(\mathrm{HK})$, phosphofructokinse (PFK), and pyruvate kinase (PK). LDHA is a key enzyme that catalyzes the conversion of pyruvate to lactate. Metformin also decreased LDHA, which according to knock-down studies in BC cell lines, may reduce proliferative rates by switching cellular mitochondria to oxidative phosphorylation [66]. Additionally, metformin treatment inhibited pyruvate dehydrogenase kinase (PDK), which facilitates the conversion of pyruvate to acetyl Co-A.

We postulate that these major shifts in glucose transport and glycolysis are a major mechanism by which metformin reduces growth, oncogenesis, induces apoptosis and may enhance sensitivity to other chemotherapeutic agents. While we have little data from our lab that suggests the latter, data from others provides a rationale for this view. Reduction of LDH by genetic manipulation or the chemical inhibitor oxamate have reversed of taxol-resistance and induced apoptosis in $\mathrm{BC}$ cells [66].

\begin{tabular}{|c|c|c|c|c|}
\hline Gene Assignment & Gene Symbol & Ref Seq & Fold-Change (G5 Met vs. G5) & P-Value \\
\hline \multicolumn{5}{|c|}{ GLUCOSE TRANSPORTERS } \\
\hline Solute Carrier Family 2 Member 1 (Facilitated Glucose Transporter) & SLC2A1 & NM_006516 & -1.71564 & $1.88 \mathrm{E}-05$ \\
\hline Solute Carrier Family 2 Member 10 (Facilitated Glucose Transporter) & SLC2A10 & NM_030777 & -1.8308 & 0.000166115 \\
\hline Solute Carrier Family 6 Member 14 (Amino Acid Transporter) & SLC6A14 & NM_007231 & -2.44598 & 2.17E-06 \\
\hline Solute Carrier Family 37 Member 4 (Glucose-6-Phosphate Transporter) & SLC37A4 & NM_001164277 & -2.38101 & 4.34E-06 \\
\hline Solute Carrier Family 2 Member 12 (Facilitated Glucose Transporter) & SLC2A12 & NM_145176 & 1.5 & 4.25E-05 \\
\hline \multicolumn{5}{|c|}{ GLUCOSE METABOLISM } \\
\hline \multicolumn{5}{|l|}{ Glycolysis } \\
\hline Enolase 1 (alpha) & ENO1 & NM_001428 & -1.5703 & 7.57E-07 \\
\hline Glucose-6-Phosphate Isomerase & GPI & NM_000175 & -1.64235 & 1.76E-05 \\
\hline
\end{tabular}


Citation: Wahdan-Alaswad RS, Edgerton SM, Salem HS, Thor AD (2018) Metformin Targets Glucose Metabolism in Triple Negative Breast Cancer . J Oncol Transl Res 4: 129. doi:10.4172/2476-2261.1000129

Page 4 of 6

\begin{tabular}{|c|c|c|c|c|}
\hline Phosphofructokinase, Liver & PFKL & NR_024108 & -1.51114 & 2.73E-05 \\
\hline Phosphoglycerate Kinase 1 & PGK1 & NM_000291 & -2.49786 & $7.32 \mathrm{E}-08$ \\
\hline Phosphoglucomutase 1 & PGM1 & NM_002633 & -1.55441 & $6.65 \mathrm{E}-05$ \\
\hline Triosephosphate Isomerase 1 & TPI1 & NM_000365 & -1.52868 & 0.001659 \\
\hline Glucose-6-Phosphatase Catalytic Subunit 3 & G6PC3 & NM_138387 & -2.05414 & $6.25 \mathrm{E}-05$ \\
\hline 6-Phosphofructo-2-Kinase/Fructose-2,6-Biphosphatase 4 & PFKFB4 & NM_004567 & -2.2822 & 1.12E-06 \\
\hline Transketolase & TKT & NM_001135055 & 1.52762 & $3.90 \mathrm{E}-05$ \\
\hline Pyruvate Kinase, Muscle & PKM2 & NM_182470 & -1.72714 & 4.15E-06 \\
\hline Lactate Dehydrogenase A & LDHA & NM_005566 & -1.86317 & $1.22 \mathrm{E}-08$ \\
\hline Phosphoglucomutase 5 & PGM5 & NM_021965 & 1.51945 & 0.001669 \\
\hline Phosphoglucomutase 5 Pseudogene 2 & PGM5P2 & NR_002836 & 1.7348 & 0.000269 \\
\hline Aldo-Keto Reductase Family 1 Member B10 & AKR1B10 & NM_020299 & 2.9025 & $1.08 \mathrm{E}-06$ \\
\hline Aldo-Keto Reductase Family 1 Member C2 & AKR1C2 & NM_001354 & 2.63487 & 1.44E-06 \\
\hline Pyruvate Dehydrogenase Kinase 3 & PDK3 & NM_005391 & -2.41605 & $1.79 \mathrm{E}-05$ \\
\hline Pyruvate Dehydrogenase Kinase 4 & PDK4 & NM_002612 & 4.13308 & 2.07E-06 \\
\hline Pyruvate Dehyrogenase Phosphatase Catalytic Subunit 1 & PDP1 & NM_001161778 & 2.83315 & $5.78 \mathrm{E}-06$ \\
\hline Prenyl (Decaprenyl) Diphosphate Synthase Subunit 1 & PDSS1 & NM_014317 & -2.09161 & $8.58 \mathrm{E}-05$ \\
\hline Phosphoglycerate Mutase 1 (Brain) & PGAM1 & NM_002629 & -2.54865 & $5.42 \mathrm{E}-07$ \\
\hline Phosphoglycerate Mutase Family Member 4 & PGAM4 & NM_001029891 & -2.26653 & 2.89E-06 \\
\hline UDP-Glucose 6-Dehydrogenase & UGDH & NM_003359 & -2.0062 & 3.93E-05 \\
\hline \multicolumn{5}{|l|}{ Gluconeogenesis } \\
\hline Pyruvate Carboxylase & PC & NM_001040716 & -2.39828 & $7.29 \mathrm{E}-06$ \\
\hline \multicolumn{5}{|l|}{ Tricarboxylic Acid Cycle (TCA) } \\
\hline ATP Citrate Lyase & ACLY & NM_001096 & -4.25837 & 2.97E-10 \\
\hline Aconitase 1 & ACO1 & NM_002197 & -1.56798 & 0.001286 \\
\hline Dihydrolipoamide S-Acetyltransferase & DLAT & NM_001931 & -1.76732 & $1.98 \mathrm{E}-07$ \\
\hline Isocitrate Dehydrogenase 2 (NADP+) & IDH2 & NM_002168 & -1.75573 & $6.09 \mathrm{E}-06$ \\
\hline Oxoglutarate (Alpha-Ketoglutarate) Dehydrogenase (Lipoamide) & OGDH & NM_002541 & -1.78156 & 0.000117 \\
\hline Pyruvate Dehydrogenase (Lipoamide) & PDHA1 & NM_000284 & -1.61653 & 1.82E-05 \\
\hline Succinate Dehydrogenase Complex Subunit A (Flavoprotein) & SDHA & NM_004168 & -1.64205 & 2.66E-05 \\
\hline Succinate Dehydrogenase Complex Subunit C (Integral Membrane Protein) & SDHC & NM_003001 & -1.59586 & 5.46E-05 \\
\hline
\end{tabular}

Table 1: Metformin attenuated the expression of a number of important genes involved in glucose metabolism. Over 20 genes were shown to be down-regulated.

\section{Material and Methods}

Cell line treatment and gene profile array were previously described $[27,58]$.

\section{Gene expression microarray}

Microarray analysis of MDA-MB-468 cells cultured in media with 5 $\mathrm{mM}$ glucose in the presence or absence of $10 \mathrm{mM}$ metformin. mRNA from treated cells was purified and analyzed using Affymetrix Human Gene 1.0 ST Array platform. Genes differentially down-regulated by 
metformin are highlighted in red. A biological triplicate experimental design was used to determine standard error.

\section{Statistics}

Statistical considerations and calculations of metformin-mediated inhibition of cholesterol pathway genes were performed using Graph Pad Prism 7 software. Statistical analysis of the experimental data was performed using a 2-sided Student t-test. Significance was set at a $\mathrm{P}<0.05$ values. Gene array samples are representative of biological replicates.

\section{Conclusions}

Metformin is the only anti-diabetic agent with anti-cancer activity, whereas other agents used in patients with this disease or metabolic syndromes have been shown to increase cancer incidence and reduce cancer-associated survival. Metformin has broad effects on multiple targets of the dysregulated lipid and carbohydrate metabolism associated with $\mathrm{BC}$, and more specifically, TNBC. In this report, we demonstrate that metformin specifically reduces the expression of key glucose transporters in TNBC, including GLUT1. We also show that it reduces transcription of key enzymes in the glycolytic pathway that are critical for cancer replication and survival. Because of the increasing prevalence of obesity, the relationships between excess body weight and cancer development and the underlying biological mechanisms need to be further investigated to prevent and treat $\mathrm{BC}$ in the future.

\section{Acknowledgment}

Support for the UCDenver Micro Array Core Facility is provided in part by the Genomics Shared Resource of Colorado's NIH/NCI Cancer Center Support Grant P30CA046934. Support for the UCDenver Cancer Center DNA Sequence and Analysis Shared Resource Core for cell line authentication is provided in part by Colorado's NIH/NCI Cancer Center Support Grant P30CA043934.

\section{Grant Support}

Grant support provided in part by Susan G Komen for the Cure K100575 to RSW, ZF, SME, and ADT; ACS-IRG 16-184-56 RSW from the American Cancer Society; CCL-C92110 RSW and ADT from the Colorado Cancer League.

\section{References}

1. American Cancer Society: Cancer Facts and Figures 2018 (2018). Atlanta, Ga.

2. Falato C, Tobin NP, Lorent J, Lindstrom LS, Bergh J, et al. (2016) Intrinsic subtypes and genomic signatures of primary breast cancer and prognosis after systemic relapse. Mol Oncol 10: 517-525.

3. Lehmann BD, Bauer JA, Chen X, Sanders ME, Chakravarthy AB, et al. (2011) Identification of human triple-negative breast cancer subtypes and preclinical models for selection of targeted therapies. J Clin Invest 121: 2750-2767.

4. Kerlikowske K, Gard CC, Tice JA, Ziv E, Cummings SR, et al. (2016) Risk Factors That Increase Risk of Estrogen Receptor-Positive and -Negative Breast Cancer. J Natl Cancer Inst 109.

5. Suba Z (2014) Triple-negative breast cancer risk in women is defined by the defect of estrogen signaling: preventive and therapeutic implications. Onco Targets Ther 7: 147-164.

6. Singletary SE (2003) Rating the risk factors for breast cancer. Ann Surg 237: 474-482.
7. McPherson K, Steel CM, Dixon JM (2000) ABC of breast diseases. Breast cancer-epidemiology, risk factors, and genetics. BMJ 321: 624-628.

8. Wolf I, Sadetzki S, Catane R, Karasik A, Kaufman B (2005) Diabetes mellitus and breast cancer. Lancet Oncol 6: 103-111.

9. Shaw RJ (2006) Glucose metabolism and cancer. Curr Opin Cell Biol 18: 598-608.

10. Schmitz KH, Neuhouser ML, Agurs-Collins T, Zanetti KA, CadmusBertram L, et al. (2013) Impact of obesity on cancer survivorship and the potential relevance of race and ethnicity. J Natl Cancer Inst 105: 1344-1354.

11. Yang XR, Sherman ME, Rimm DL, Lissowska J, Brinton LA, et al. (2007) Differences in risk factors for breast cancer molecular subtypes in a population-based study. Cancer Epidemiol Biomarkers Prev 16: 439-443.

12. Lipscombe LL, Goodwin PJ, Zinman B, McLaughlin JR, Hux JE (2008) The impact of diabetes on survival following breast cancer. Breast Cancer Res Treat 109: 389-395.

13. Hall IJ, Newman B, Millikan RC, Moorman PG (2000) Body size and breast cancer risk in black women and white women: the Carolina Breast Cancer Study. Am J Epidemiol 151: 754-764.

14. Millikan RC, Newman B, Tse CK, Moorman PG, Conway K, et al. (2008) Epidemiology of basal-like breast cancer. Breast Cancer Res Treat 109: 123-139.

15. Rose DP, Haffner SM, Baillargeon J (2007) Adiposity, the metabolic syndrome, and breast cancer in African-American and white American women. Endocr Rev 28: 763-777.

16. Carey LA, Perou CM, Livasy CA, Dressler LG, Cowan D et al. (2006) Race, breast cancer subtypes, and survival in the Carolina Breast Cancer Study. JAMA 295: 2492-2502.

17. Telli M (2015) Evolving Treatment Strategies for Triple-Negative Breast Cancer. J Nat Comp Canc Netw 13: 652-654.

18. La Vecchia C, Giordano SH, Hortobagyi GN, Chabner B (2011) Overweight, obesity, diabetes, and risk of breast cancer: interlocking pieces of the puzzle. Oncologist 16: 726-729.

19. Becker S, Dossus L, Kaaks R (2009) Obesity related hyperinsulinaemia and hyperglycaemia and cancer development. Arch Physiol Biochem 115: 86-96.

20. Xue F, Michels KB (2007) Diabetes, metabolic syndrome, and breast cancer: a review of the current evidence. Am J Clin Nutr 86: 823-835.

21. Young CD, Anderson SM (2008) Sugar and fat-that's where it's at: metabolic changes in tumors. Breast Cancer Res 10: 202.

22. Hilvo M, Denkert C, Lehtinen L, Muller B, Brockmoller S, et al. (2011) Novel theranostic opportunities offered by characterization of altered membrane lipid metabolism in breast cancer progression. Cancer Res 71: 3236-3245.

23. Menendez JA, Lupu R (2007) Fatty acid synthase and the lipogenic phenotype in cancer pathogenesis. Nat Rev Cancer 7: 763-777.

24. Puig T, Vazquez-Martin A, Relat J, Petriz J, Menendez JA, et al. (2008) Fatty acid metabolism in breast cancer cells: differential inhibitory effects of epigallocatechin gallate (EGCG) and C75. Breast Cancer Res Treat 109: 471-479.

25. Chajes V, Cambot M, Moreau K, Lenoir GM, Joulin V (2006) Acetyl-CoA carboxylase alpha is essential to breast cancer cell survival. Cancer Res 66: 5287-5294.

26. Rios Garcia M, Steinbauer B, Srivastava K, Singhal M, Mattijssen F, et al. (2017) Acetyl-CoA Carboxylase 1-Dependent Protein Acetylation Controls Breast Cancer Metastasis and Recurrence. Cell Metab 26: 842-855.

27. Wahdan-Alaswad RS, Cochrane DR, Spoelstra NS, Howe EN, Edgerton SM, et al. (2014) Metformin-induced killing of triple-negative breast cancer cells is mediated by reduction in fatty acid synthase via miRNA-193b. Horm Cancer 5: 374-389.

28. Wahdan-Alaswad RS, Edgerton SM, Salem HS, Thor AD (2018) Metformin Targets Cholesterol Biosynthesis Pathway, GM1 Lipid Raft Stabilization, EGFR Signaling and Proliferation in Triple Negative Breast Cancers. Canc Therapy \& Oncol Int J 9: 555765. 
29. World Health Organization (1999) Definition, Diagnosis and Classification of Diabetes Mellitus and Its Complications: Report of a WHO Consultation. Geneva, Switzerland: World Health Organization.

30. Esposito K, Gentile S, Candido R, De Micheli A, Gallo M, et al. (2013) Management of hyperglycemia in type 2 diabetes: evidence and uncertainty. Cardiovasc Diabetol 12: 81.

31. Osaki Y, Taniguchi S, Tahara A, Okamoto M, Kishimoto T (2012) Metabolic syndrome and incidence of liver and breast cancers in Japan. Cancer Epidemiol 36: 141-147.

32. Rosato V, Bosetti C, Talamini R, Levi F, Montella M, et al. (2011) Metabolic syndrome and the risk of breast cancer in postmenopausal women. Ann Oncol 22: 2687-2692.

33. Dowling RJ, Niraula S, Stambolic V, Goodwin PJ (2012) Metformin in cancer: translational challenges. J Mol Endocrinol 48: R31-43.

34. Goodwin PJ, Pritchard KI, Ennis M, Clemons M, Graham M, et al. (2008) Insulin-lowering effects of metformin in women with early breast cancer. Clin Breast Cancer 8: 501-505.

35. Goodwin PJ, Stambolic V (2011) Obesity and insulin resistance in breast cancer-chemoprevention strategies with a focus on metformin. Breast 20 Suppl 3: 31-35.

36. Goodwin PJ, Thompson AM, Stambolic V (2012) Diabetes, metformin, and breast cancer: lilac time? J Clin Oncol 30: 2812-2814.

37. Picon-Ruiz M, Morata-Tarifa C, Valle-Goffin JJ, Friedman ER, Slingerland JM (2017) Obesity and adverse breast cancer risk and outcome: Mechanistic insights and strategies for intervention. CA Cancer J Clin 67: 378-397.

38. Gezgen G, Roach EC, Kizilarslanoglu MC, Petekkaya I, Altundag K (2012) Metabolic syndrome and breast cancer: an overview. J Buon 17: 223-229.

39. Warburg O, Wind F, Negelein E (1927) The Metabolism of Tumors in the Body. J Gen Physiol 8: 519-530.

40. Lien EC, Lyssiotis CA, Cantley LC (2016) Metabolic Reprogramming by the PI3K-Akt-mTOR Pathway in Cancer. Recent Results Cancer Res 207 39-72.

41. Lin DS, Kao SH, Ho CS, Wei YH, Hung PL, et al. (2017) Inflexibility of AMPK-mediated metabolic reprogramming in mitochondrial disease. Oncotarget 8: 73627-73639.

42. Shen L, O'Shea JM, Kaadige MR, Cunha S, Wilde BR (2015) Metabolic reprogramming in triple-negative breast cancer through Myc suppression of TXNIP. Proc Natl Acad Sci USA 112: 5425-5430.

43. Chen C, Tang Q, Zhang Y, Dai M, Jiang Y, et al. (2017) Metabolic reprogramming by HIF-1 activation enhances survivability of human adipose-derived stem cells in ischaemic microenvironments. Cell Prolif 50 .

44. Graveel CR, DeGroot JD, Su Y, Koeman J, Dykema K, et al. (2009) Met induces diverse mammary carcinomas in mice and is associated with human basal breast cancer. Proc Natl Acad Sci USA 106: 12909-12914.

45. Lanning NJ, Castle JP, Singh SJ, Leon AN, Tovar EA, et al. (2017) Metabolic profiling of triple-negative breast cancer cells reveals metabolic vulnerabilities. Cancer Metab 5: 6 .

46. Robey RB, Hay N (2009) Is Akt the "Warburg kinase"?-Akt-energy metabolism interactions and oncogenesis. Semin Cancer Biol 19: 25-31.

47. Gottlieb E, Vousden KH (2010) p53 regulation of metabolic pathways. Cold Spring Harb Perspect Biol 2: 001040

48. Godoy A, Ulloa V, Rodriguez F, Reinicke K, Yanez AJ, et al. (2006) Differential subcellular distribution of glucose transporters GLUT1-6 and GLUT9 in human cancer: ultrastructural localization of GLUT1 and GLUT5 in breast tumor tissues. J Cell Physiol 207: 614-627.

49. Vander Heiden MG, Locasale JW, Swanson KD, Sharfi H, Heffron GJ, et al. (2010) Evidence for an alternative glycolytic pathway in rapidly proliferating cells. Science 329: 1492-1499.
50. Wu W, Zhao S (2013) Metabolic changes in cancer: beyond the Warburg effect. Acta Biochim Biophys Sin (Shanghai) 45: 18-26.

51. Huang WC, Hsu SC, Huang SJ, Chen YJ, Hsiao YC, et al. (2013) Exogenous expression of human SGLT1 exhibits aggregations in sodium dodecyl sulfate polyacrylamide gel electrophoresis. Am J Transl Res 5: 441-449.

52. Krzeslak A, Wojcik-Krowiranda K, Forma E, Jozwiak P, Romanowicz H, et al. (2012) Expression of GLUT1 and GLUT3 glucose transporters in endometrial and breast cancers. Pathol Oncol Res 18: 721-728.

53. Hussein YR, Bandyopadhyay S, Semaan A, Ahmed Q, Albashiti B, et al. (2011) Glut-1 Expression Correlates with Basal-like Breast Cancer. Transl Oncol 4: 321-327.

54. Wellberg EA, Johnson S, Finlay-Schultz J, Lewis AS, Terrell KL, et al. (2016) The glucose transporter GLUT1 is required for ErbB2-induced mammary tumorigenesis. Breast Cancer Res 18: 131.

55. Petrocca F, Altschuler G, Tan SM, Mendillo ML, Yan H, et al. (2013) A genome-wide siRNA screen identifies proteasome addiction as a vulnerability of basal-like triple-negative breast cancer cells. Cancer Cell 24: 182-196.

56. Zhao Y, Shen L, Chen X, Qian Y, Zhou Q (2015) High expression of PKM2 as a poor prognosis indicator is associated with radiation resistance in cervical cancer. Histol Histopathol 30: 1313-1320.

57. Cappelletti V, Iorio E, Miodini P, Silvestri M, Dugo M, et al. (2017) Metabolic Footprints and Molecular Subtypes in Breast Cancer. Dis Markers 2017: 7687851.

58. Wahdan-Alaswad R, Fan Z, Edgerton SM, Liu B, Deng XS, et al. (2013) Glucose promotes breast cancer aggression and reduces metformin efficacy. Cell Cycle 12: 3759-3769.

59. Alimova IN, Liu B, Fan Z, Edgerton SM, Dillon T, et al. (2009) Metformin inhibits breast cancer cell growth, colony formation and induces cell cycle arrest in vitro. Cell Cycle 8: 909-915.

60. Liu B, Fan Z, Edgerton SM, Deng XS, Alimova IN, et al. (2009) Metformin induces unique biological and molecular responses in triple negative breast cancer cells. Cell Cycle 8: 2031-2040.

61. Liu B, Fan Z, Edgerton SM, Yang X, Lind SE, Thor AD (2011) Potent antiproliferative effects of metformin on trastuzumab-resistant breast cancer cells via inhibition of erbB2/IGF-1 receptor interactions. Cell Cycle 10: 2959-2966.

62. Checkley LA, Rudolph MC, Wellberg EA, Giles ED, Wahdan-Alaswad RS, et al. (2017) Metformin Accumulation Correlates with Organic Cation Transporter 2 Protein Expression and Predicts Mammary Tumor Regression In Vivo. Cancer Prev Res (Phila) 10: 198-207.

63. Giles ED, Wellberg EA, Astling DP, Anderson SM, Thor AD, et al. (2012) Obesity and overfeeding affecting both tumor and systemic metabolism activates the progesterone receptor to contribute to postmenopausal breast cancer. Cancer Res 72: 6490-6501.

64. Deng XS, Wang S, Deng A, Liu B, Edgerton SM, et al. (2012) Metformin targets Stat 3 to inhibit cell growth and induce apoptosis in triple-negative breast cancers. Cell Cycle 11: 367-376.

65. Wahdan-Alaswad R, Harrell JC, Fan Z, Edgerton SM, Liu B, et al. (2016) Metformin attenuates transforming growth factor beta (TGF-beta) mediated oncogenesis in mesenchymal stem-like/claudin-low triple negative breast cancer. Cell Cycle 15: 1046-1059.

66. Valvona CJ, Fillmore HL, Nunn PB, Pilkington GJ (2016) The Regulation and Function of Lactate Dehydrogenase A: Therapeutic Potential in Brain Tumor. Brain Pathol 26: 3-17. 